\title{
Heuristic for brand artefacts co-design: a computer-mediated proposal
}

\author{
Catarina Lélis and Óscar Mealha
}

\begin{abstract}
Brands undeniably constitute a significant asset for a company and are usually specified in its organisational strategy. Typically, employees are not invited to participate in debates or decisions about branding since they access brand information in a unidirectional way. Many of them do not even know how to convey appropriate messages and fail to recognize the importance of brand consistency. In this paper we propose a heuristic for an interaction structure aimed at brand artefact co-design, through a computer-mediated platform, so that employees may learn about the brand, develop brand knowledge, propose meaningful brand artefacts and manage the evaluation of their peers' proposals. We prepared a non-functional prototype of a Participatory Brand Centre and tested it in focus group sessions with the University of Aveiro's employees. We found that though our proposal is consistent with employees' brand-related needs, authorship is a sensitive issue.
\end{abstract}

Keywords: brand artefacts, co-design, employees, heuristic, computer-mediated communication

\section{Introduction}

The perception that employees have about what their organisation stands for has grown in importance for identity and image auditing, as employees are increasingly considered the main agents of organisational reputation (Ind, 2007; Ind and Bjerke, 2007). On the other hand, employees can only defend and use the brand properly if they have a deeper understanding of it. Employees must know the organisational identity of their company so that they can play a committed role in how they communicate and expose it to others (Ackerman, 2000; Punjaisri and Wilson, 2007; King and Grace, 2008).

Moreover, as communication elements, brands no longer fit the historical model of information transmission proposed by Shannon and Weaver, particularly since the one-way approach has been (or is being) supplanted, as several scholars and experts have advocated (Arnhold, 2010; Burmann and Zeplin, 2005; Harris, 2007; Ind and 
Bjerke, 2007; Merz, He and Vargo, 2009; Neumeier, 2006; Schultz and Hatch, 2006). Such re-orientation requires a new kind of organisational philosophy, which must be established and assimilated by everyone within the organisation: aside from the fact that all human resources should be minimally familiar with the brand identity, they should also realise that their participation in the brand leads to the co-creation and conversion of existing knowledge, and may potentially represent added value, through meaningful contributions - which may emerge from experiencing brand artefacts and engagement in internal branding activities (Ind, 2007).

We argue that brand identity and brand experience should be relevant subjects for all employees, who should access brand information and externalise their brand knowledge to convey appropriate messages and to use the brand in a consistent way.

In this paper we propose a $B a$ for brand artefact co-design and a heuristic for brand experience and co-creation of brand artefacts, with preliminary assumptions based on a thorough literature review and confirmed in focus groups conducted at the University of Aveiro, which recently had its brand design changed, leading to inconsistencies in the application and use of the brand.

\section{Literature review}

In their seminal work, Nonaka and Takeuchi (1995) propose two kinds of knowledge (explicit and tacit) and advocate knowledge as a critical success factor for organisations. The main purpose of knowledge management is to encode and convert individual knowledge into organisational assets, as elements of the organisational identity that can be shared with other individuals, who may, in turn, work on and modify them (idem).

However, within organisations, the idea of knowledge sharing and ownership is not that simple. Jarvenpaa and Staples (2001) explain that individuals only share their knowledge with workmates when they consider that knowledge to be the property of the 
whole organisation, i.e. collective knowledge that can be applied in collaborative activities and, thus, necessarily shared. The domain of a social identity affecting the whole organisation seems to bring with it clear benefits for knowledge integration. Therefore, identity is something inherited, generated by the continuous accumulation and renewal of knowledge. A properly managed identity system would be the first step towards the creation, conversion and transfer of brand knowledge, embodied in identity artefacts of varying tangibility (Masino and Zamarian, 2003; Moingeon and Ramanantsoa, 1997; Morgan, 2006; Rafaeli and Pratt, 2006; Schein, 2010; Schultz, Hatch and Ciccolella, 2006; Vilnai-Yavetz and Rafaeli, 2006) and, consequently, of varying explicitness. Organisational artefacts, which are identity vehicles, are likely to communicate identity more effectively to internal audiences, allowing all employees to collectively assume that identity. Thus, organisational identity is strengthened by the conversion and integration of collective knowledge. Such is also the case with brand identity. Brand identity consists of a set of tangible and intangible organisational artefacts, symbolic, functional and aesthetic products (Vilnai-Yavetz and Rafaeli, 2006) that are conceptualized and implemented in order to explain organisational methods, behaviours and routines. They are essential to brand design activities.

\section{Employee participation}

Involving employees in activities whereby they participate in joint decision-making situations that Buur (2013) describes, applied to business issues - allows them to be innovative and consolidate the information shared, as well as experience the brand (which is useful for their own brand alignment). Under these circumstances, employees get a better appraisal of their role and commit to the brand promise, which they must represent and assume (Chong, 2007), enabling them to perform actions that will enrich the brand (McEnally and De Chernatony, 1999). Indeed, brand knowledge is a 
significant factor in employees' participation in internal branding activities (Mangold and Miles, 2007), particularly when they are expected to co-design, in an act of collective creativity (Sanders and Stappers, 2008).

Schultz, Hatch and Ciccolella (2006) advocate the importance of giving employees the opportunity to explore, experience and use organisational and brand artefacts, thus allowing them to process and enhance the meanings of these artefacts and eventually produce co-designed contributions with different and new meanings. Furthermore, Thorpe and Gamman clearly state that 'Designers do not have the control over resources that would allow them to set and fulfil their own agendas; hence they routinely deliver design that serves the interests of the market over those of society: shareholders over stakeholders' $(2011,220)$. Accordingly, Feast (2012) argues that designers should exploit and take avail of the knowledge of different stakeholders in order to develop a broader and shared knowledge, enabling organisations to become better prepared at dealing with society's wicked-problems.

Academics and practitioners also welcome this participatory design focus: designer Marty Neumeier's perspective is that brands are inherently flexible so they should be considered as constantly evolving. He welcomes interventions from nonspecialised individuals, provided a brand's most basic and explicit design attributes are not lost nor violated (2006). Fischer and Scharff (2000) discuss the relevance of engaging workers in design activities through meta-design approaches. Fischer (2007) states that meta-design's basic assumption is that not every possible use and problem can be fully anticipated at the moment of conception and creation (design time). Only after implementation (use time) can users discover mismatches between their needs and what the artefact actually provides. Thus, meta-design overcomes this limitation by allowing users to contribute towards the functionalities, meanings and contents of 
systems, which are set to remain in a state of constant development because they cannot be defined, once and forever, in design time (Bjögvinsson, Ehn and Hillgren, 2012).

So it is, again, with brands. Therefore, organisations should make available appropriate infrastructures to facilitate brand co-design and the co-creation of brand knowledge.

\section{A Ba for brand artefacts co-design}

$B a$ is a Japanese term that represents a context in which 1) it is possible to openly interpret and assign meanings to information; 2) individuals can reflect on their own and others' experiences and 3) new knowledge is subsequently created. Ikujiro Nonaka adapted this concept within the management framework to be conceived as a "space" for sharing and for the creation of knowledge, which can occur individually, at informal meetings, in moments of team-work, in classrooms, in emails circulating via multiple entities, or even through contact with customers or other stakeholders, in a given organisational context. It can be physical, virtual, mental or any combination of the above (Nonaka and Konno, 2005). According to these authors, to participate in a $B a$, individuals must overcome their own limited perspectives and physical boundaries, so that they can be effectively creative.

Sharp ${ }^{\mathrm{TM}}$ created a $B a$ to accumulate a customer knowledge-base which allows project teams to develop new product concepts and proposals. In addition, by managing several $B a$ in their stores, and by continuously building and testing hypotheses, 7Eleven Japan ${ }^{\mathrm{TM}}$ successfully creates knowledge: local store employees accumulate tacit knowledge and market feelings (which they cannot explain easily) about customer needs through long-term experiences in dealing with them. Such knowledge becomes explicit when these employees, even part-time workers, are given the responsibility to order products based on their insights (Nonaka, Konno and Toyama, 2001). 
Furthermore, according to meta-design assumptions, a $B a$ should be flexible and scalable, adapting as participants learn. It is also possible and desirable to take the Web (with its social positioning) as a primordial means of communication, as it allows the expansion of creativity, both individual and collective, decentralising the co-design process (Fischer, 2007).

Neto \& Choo (2010) suggest that the use of information and communication technologies (ICT) can increase the scale and reach of knowledge creation across an organisation and the efficiency of how that knowledge is shared and accessed by employees. All computer-mediated communication (CMC) activities that capture, store and provide efficient access to knowledge (Alavi and Leidner, 2001; Tee, 2005) are defined by their interactive context, which allows the sharing of beliefs and the expression of new ideas. In a recent study of communication within collective innovation communities, Paulini, Murty and Maher $(2013,90)$ highlight that 'When individuals from a diversity of backgrounds become involved in online innovation, their collective focus can be used to address complex problems that benefit from multiple perspectives'.

Therefore, a $B a$ enables the accumulation of knowledge resources and assets (information) such that they may be integrated in a cyclical, interactive and ecological manner. We believe that a $B a$ for brand artefact co-design should be based on three fundamental criteria:

- Individual or collective/shared brand-related learning experiences;

- Opportunities for participation and for creative and sustainable brand exploration;

- Employees are allowed to perform additional roles, beyond those usually assigned by labour contracts. 
The $B a$ used in this research is essentially supported by an online community, open to the overall workforce, in which power, control, creativity and responsibility are distributed. Thus, our research question is: 'What structure should support a $B a$ for brand artefact co-design?'

\section{Proposal for an interaction structure}

We believe that a $B a$ for brand artefact co-design should be governed by three domains of interaction (Figure 1):

- Mentoring towards brand knowledge;

- Co-design and Transfer (C\&T) of brand artefacts;

- Evaluation of brand artefacts.

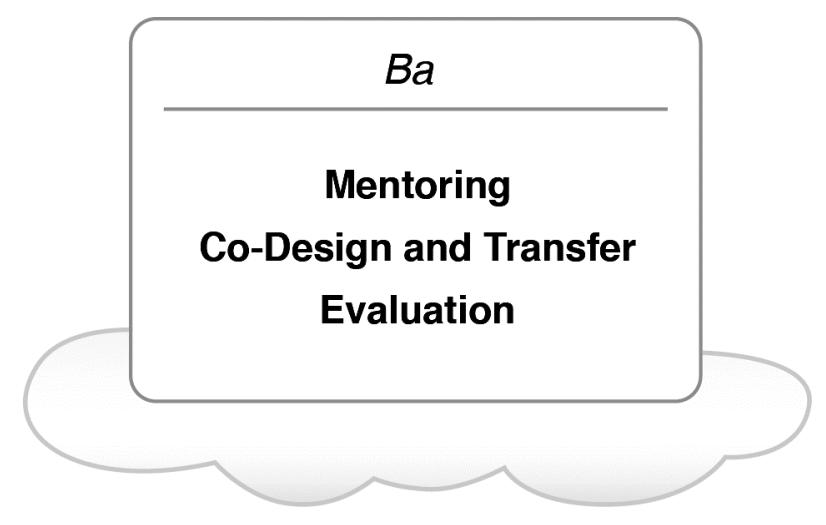

Figure 1. - The three main domains of the interaction structure for brand artefact codesign

In these domains the user can manage brand information in either or both of the following two ways: 1) sequentially, beginning with Mentoring, which should provide conditions for C\&T, and in turn allows the user to evaluate; 2 ) in a non-sequential or irregular manner, so that the user can choose how to experience the brand (Figure 2). 


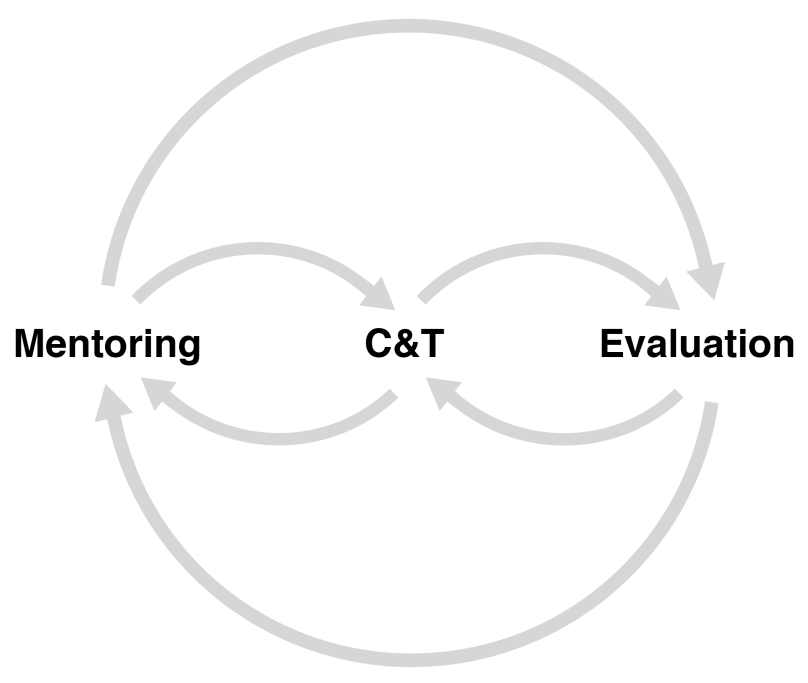

Figure 2. - The iterative structure of the $B a$ for brand artefact co-design

Indeed, it is the combination of these two approaches that defines the 'eternally mutant' principle, which characterises artefacts built under co-design, involving the participation of many individuals, over diverse and differentiated interventions, during design time and use time.

\section{Mentoring towards brand knowledge}

Besides their own overall understanding of the brand and what it stands for, it is essential that all participants 1) have access to basic information about the strategy underlying the brand; 2) understand the brand design standards and 3) are endowed with sufficient visual literacy to be able to represent their own ideas and interpretations of the brand, without damaging it. It is, therefore, a process of orientation of brand knowledge, which involves observation and retention of information, but above all, trial and error, especially when converting tacit into explicit knowledge. In short, it is about a learning process, since 'learning is a participatory process that involves doing, becoming and belonging, not simply acquiring' (Barab et al., 2001, 76). 
Fischer (2009) proposes that both educational and labour environments should be reconceptualised, becoming spaces in which individuals are both contributors and learners and wherein peer-to-peer learning is supported by fellows who do not see themselves as teachers, but as mentors/facilitators. Such new learning models are strongly associated with the use of ICT, which minimise learning efforts and ensure better access to resources and to a larger number of interactions (McLoughlin and Lee, 2007). In this light, interactions have a crucial and decisive role in learning and knowledge development.

Learning the brand is thus more related to a process of connecting than of acquisition and, should consequently be oriented along these lines. Such guidance can be provided by individuals (who may be specialised peers or outsourced professionals performing the role of mentors, such as designers) or mediated by CMC systems, endowed with algorithmic capacities, appropriate for this purpose. However, as proposed by Punjaisri and Wilson (2007), who identify learning programs and ICT as the main mechanisms used in internal branding activities, guidance can also be provided by these two agents combined - mentors supported by CMC systems — which, in the present research context, we consider the preferred scenario.

We advocate that when learning and communication processes are computermediated, individuals foster a better understanding of the community in which they participate, influenced by the interactions they experience and by their own interpretation of such interactions. Therefore, we suggest that it is essential to provide the tools best suited to such exchanges, so as to enable the co-creation and management of brand knowledge (Lélis and Mealha, 2012, 2013, 2014).

\section{Co-Design and Transfer (C\&T) of brand artefacts}

Considering that the main goal of meta-design is to allow anyone to act as a designer, 
human resources should, in the appropriate circumstances, be given the opportunity to freely experience brand artefacts in situations relevant to themselves. In addition, they should participate in activities aimed at co-designing brand artefacts, where appropriation and gamification are key skills. According to Jenkins (2009), appropriation is the ability to extract content from a given context and to recontextualise it again, giving it new meanings. Gamification is widely recognized as a way of motivating individuals to learn different contents from those they regularly deal with; it is also a means of exploring and processing not only knowledge but also decision-making (Zichermann and Cunningham, 2011). Accordingly, two of the purposes of design games identified by Vaajakallio and Mattelmäki $(2014,64)$ are of particular relevance to our $B a$ proposal: 1) 'empowering (...) people who are not traditionally part of design processes but are influenced by design: to provide hands-on tools for establishing a common language between designers and users and to involve users in discussions on existing and future alternatives' and 2) for the engagement of 'diverse stakeholders in expressing, negotiating and generating a shared understanding of users, use contexts and technology in early concept design' (idem).

The meaning of a brand is produced by those who intellectually, emotionally and aesthetically engage with brand-related artefacts, words or events, so that these explicit symbols represent tacit knowledge — ideas, feelings or experiences (Hatch, 2007). Ind (2007) states that one of the benefits of involving employees in defining the brand and its artefacts is that it is highly likely that such an experiential process will become a cathartic one, as it encourages them to reflect on the nature of the brand, to understand its attributes and assess the whole range of meanings arising from the contributions of all. 
We can conceive the design and subsequent sharing of brand artefacts by adopting these same assumptions (Lélis and Mealha, 2012, 2013, 2014). Thus, the brand 'code' becomes accessible to every employee through a framework that withdraws brand design from the traditional and closed perspective in which 'a corporation produces a brand's physical embodiment, exclusively narrates the brand's text, unilaterally directs the brand experience, and broadcasts the brand's meaning' (Pitt et al., 2006, 119) to a diametrically opposed and open perspective, in which individuals perform as co-designers.

\section{Evaluation of brand artefacts}

The validity and conformity of these contributions within a CMC platform are evaluated at several moments.

The first moment will be supported and mediated by the CMC system so that it can occur synchronously. It is assumed that participants will strive to make their ideas clear and will seek to represent them using a common and explicit code to express all essential brand values and their physical characteristics, being encouraged (but not enforced) to comply with the rules regulating the brand. The CMC platform should thus help participants to experience the brand, while acknowledging these precise norms and guidelines.

The second moment of evaluation takes place among the community as a whole, wherein the group of participants must collectively be given an opportunity to comment on their peers' contributions, providing relatively quick feedback. Simple voting scales also allow employees to easily criticise and classify the brand artefacts that others have created (taking into consideration overall brand appreciation). The aggregation of brand-related information and individual tacit brand knowledge facilitates the 
involvement of human resources in evaluating brand artefacts proposed by their peers, thus harnessing their 'collective wisdom' (Surowiecki, 2005).

The third instance of evaluation aims to validate any brand artefacts that employees may propose. A participatory approach leads to a reduction in control over the brand so Reichwald, Möslein and Piller (2008) propose the appointment of an individual to act as gatekeeper, who will perform a screening role. Following the second moment of evaluation, the gatekeeper will thus submit the top ranked brand artefacts for the consideration of senior management. A single individual, who is firmly responsible for the brand, usually assumes this role.

\section{Research methodology}

To validate the proposed structure it was important to choose an institutional universe in which a deeper and empirical analysis could be made. The aim was to address, primarily, the participation needs of employees working in service provider organisations, such as universities. A case study was conducted in the academic context of the University of Aveiro (UA).

The broad approach followed was an inductive one, aimed at understanding the social context through the interpretation of participants. Academics are especially rich sources of tangible ideas for the brands that represent their universities: they establish numerous contacts, present research at conferences worldwide, exchange artefacts and souvenirs, and collect items of merchandise from the many different meetings in which they participate. Typically, they are critically minded and like to have their opinion heard at the institutional level. Therefore, they seemed an interesting public for this study. Moreover, UA recently changed its brand design (logotype and signage system), leading to inconsistent usages and somewhat unacceptable brand applications by employees, both teachers and non-teaching staff. Furthermore, UA was chosen due to 
its vast IT infrastructure, which ranges across all university areas and services. From a technological standpoint, UA is well prepared and its employees are accustomed to using ICT in all their varied functions, and they know and recognise most of the interaction paradigms associated with these technologies.

Five focus groups were set up and the participants (teachers and non-teaching staff) were invited to discuss the subject. They were shown a non-functional prototype of a Participatory Brand Centre (PBC), with the purpose of validating the proposed $B a$. The main objectives guiding the focus groups were to assess the willingness/motivation to participate in activities that may enhance UA's brand, and to present the mid-fidelity $\mathrm{PBC}$ in this institutional context, so as to raise comments and/or suggestions.

Given the importance of achieving a relaxed atmosphere in each group, and considering that the number of teachers and non-teaching staff in UA are distributed in a ratio of three to two, we organised participants in three groups of teachers and two groups composed of non-teachers, taking into account their professional categories and time within the Institution. We had the participation of 17 teachers and 15 non-teaching staff. Out of a total of 32 individuals, 18 were female and 14 were male.

The planning of the focus groups (FGs) included the creation of a flexible yet comprehensive script, to ensure that the selected technique would answer the research question, but also as a way to ensure minimum consistency between FGs (Krueger, 1994; Gaskell, 2000; Casey and Krueger, 2010; Morgan, 1997).

Besides the essential presence of a moderator, each session also included a technical observer, who took control of audiovisual equipment. Recordings of all the FGs were fully transcribed and sent to participants for validation. The meeting rooms were prepared previously, with seating in a $U$ formation so that participants could see each other during the whole session. After a brief introduction and topics review, the 
first themes to be discussed were of a general nature and easy approach, to enable the immediate participation of all (Morgan, 1997). Only then did we introduce more specific questions that prompted some reflection. Finally, the presentation and discussion of the PBC prototype took place.

\section{Findings}

Content analysis of the collected data was achieved with the support of WebQDA, a web-based software for qualitative data analysis. The overall content analysis process is visually depicted in Figure 3. After careful reading of the five transcripts, it was possible to highlight common themes, together with themes that had already been anticipated (Krueger and Casey, 2010).

We intersected our predefined analysis codes with the three domains that define the interaction structure of the $B a$ for brand artefact co-design (Table 1). The focus of analysis was much more on the meaning of generated content and on its proximity to the theoretical framework and inductions developed previously. At the same time, we considered the frequency of terms or topics to verify the relevance of our selections.

Table 1. Detail Questions and Analysis Codes.

\begin{tabular}{|l|c|}
\hline \multicolumn{1}{|c|}{ Guidance Question and Detail Questions } & Analysis Codes \\
\hline Would it make sense to implement an online resource based on the presented? & \\
\hline What is the possibility of implementing a PBC in University of XXX? & PBC Implementation \\
\hline What would be the advantages for University of XXX and its staff? & Unnecessary Features \\
\hline What features seem to be unnecessary? & Missing Features \\
\hline What features might be missing? & Obstacles \\
\hline What kind of obstacles could derail such resource? & Propensity \\
\hline Personally, what would take you to participate in such a platform? & \\
\hline
\end{tabular}




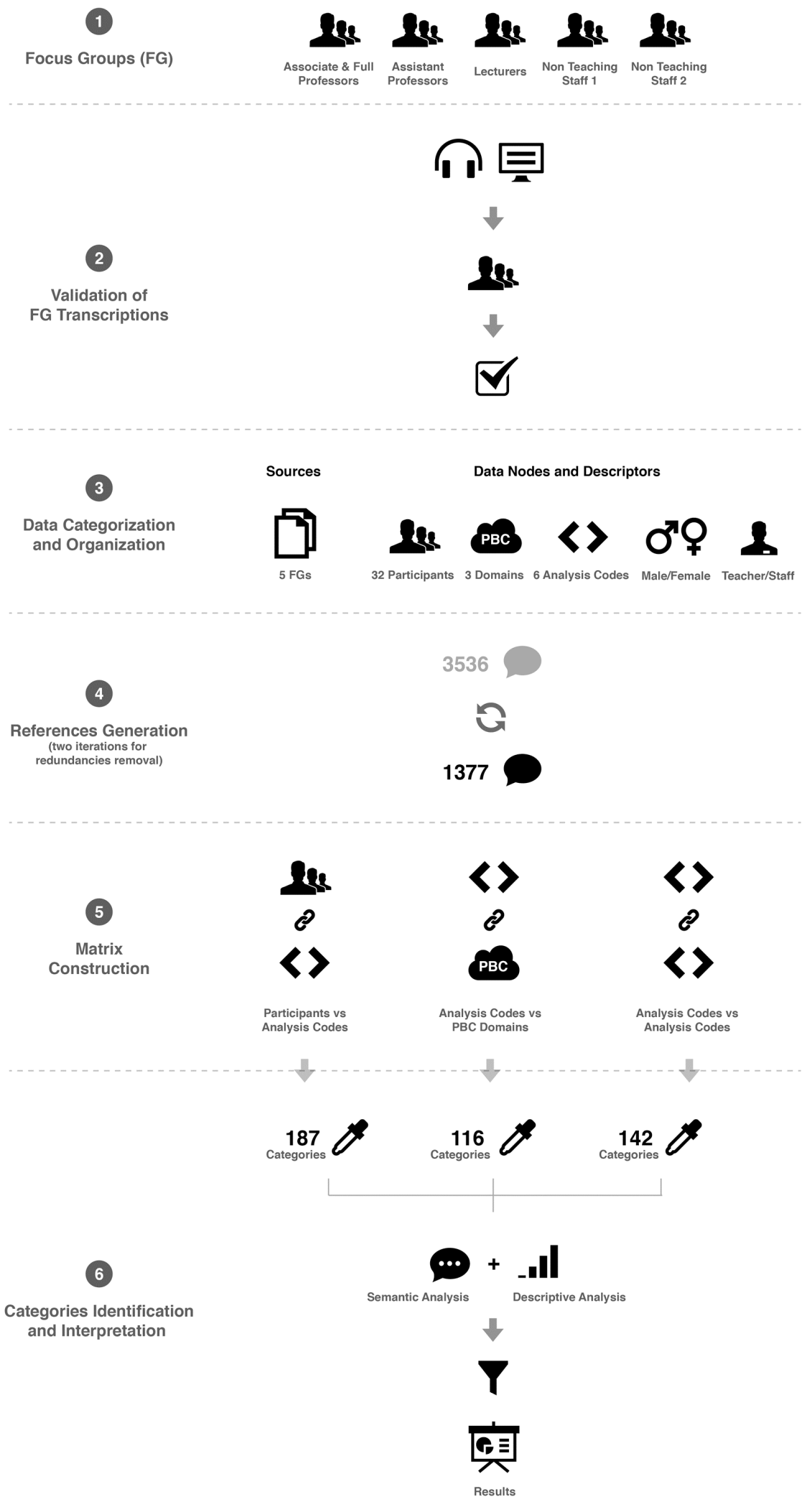

Figure 3. - The process of Focus Groups content analysis. 
This exercise allowed predefined analysis codes to be used in order to find references in their respective categories that were objectively related to each of the proposed $B a$ 's three domains (Table 1). Overall, the analysis code with the highest number of references was 'PBC Implementation', which was already expected, since it is natural that participants will share their opinions with the support of the organisational context they know.

It was in the Mentoring domain that participants found more novelty and usefulness for their work context, classifying its related features as advantages, which had a great influence on their willingness to eventually participate and use a PBC. In these cases, the most significant categories are those regarding the main technological feature, Studio, since it provides simplified access to graphic brand guidelines and a pedagogical approach. In Studio users can experience the brand's graphical, physical and explicit attributes (such as its logotype or visual symbols) through an embedded image editor, which dynamically signals incorrect brand applications, based on the brand's graphical standards.

So, I'm entering Studio and ... 'let me see... where can I find UA's logo, let me see if I'm right in using it; ok, I'm not [...] let me do that again' ... [Participant 22, Non-Teaching Staff-1 group].

Studio can be extremely useful because it allows those of us who don't know a thing about design to immediately get some evaluation of what we're doing ... I think it really would be a very cool feature! [Participant 18, Non-Teaching Staff-1 group]

Advantages are very much concerned with the possibility of a PBC being implemented at UA. However, in this regard, emphasis was given to comments that reveal a general perception of the Mentoring domain and its consequent learning aims, 
and the perceived relevance of graphic brand standards/guidelines so that participants' usage can generate satisfactory contributions, not only for the creation of professional resources, but also to generate ideas for the enhancement of the organisational brand.

I think people do not understand that if you make that green a little darker it changes everything. It's not the same brand anymore... [Participant 20, NonTeaching Staff-1 group]

Concerning the Co-design \& Transfer domain, participants see several links between the prototype presented and the possibility of implementing a PBC at the UA, revealing, explicitly and implicitly, an apparent willingness to participate in such a resource. They provided ideas associated with what they consider to be added value, suggestions of features they consider to be missing, and resources to overcome the obstacles they listed. It should be noted that one of the most common themes in this domain involves brand authorship and property, which seems to be a concern for these potential contributors:

Isn't that risky? I will be making available a set of ideas [...] isn't there any possibility of appropriation of my idea which is still in development, by someone else? [...] [Participant 1, Associates and Full Professors' group]

A place with all the graphic rules and official documents would be extremely useful [...] A place to share, also looks good, because when I share it's in this new social logic and I know there will be some kind of remix, and that someone else will pick up what I did and will adapt it. [Participant 3, Associates and Full Professors' group]

Stimulus to participation and consequentiality are also themes with expression in the $C \& T$ domain:

Probably many employees will think 'why should I be wasting my time with this PBC thing when no one ever listens to me?' [Participant 11, Assistants' group] 
Quite honestly, the PBC idea is excellent, but I fear it will tend to be inconsequential because, most certainly, top management will not get involved. [Participant 25, Non-Teaching Staff-1 group]

Regarding the Evaluation domain, participants established positive links between the prototype presented and the possibility of implementing a PBC at UA. However, in this case, there were a high percentage of references to 'Missing Features':

The Don't Like option is missing. It is for the community itself to eliminate an artefact that sucks... It would be necessary to have a more accurate algorithm like, let's say, a specific number of Don't Likes from different departments. [Participant 8, Assistants' group]

The advantages that participants identified in this domain are very much associated with brand citizenship behaviours, consequence and participation. They also acknowledge the fact that community evaluation may ease the selection of some artefacts:

OK, let's be optimistic, can you imagine? - my idea having institutional consequence... Imagine this branded chocolate bar was my idea, and that it goes along and becomes available to everyone... That's one thing I gave to the institution! [Participant 19, Non-Teaching Staff-1 group]

On the other hand, they also revealed concerns with ownership issues (regarding, again, content property):

Will this [platform] work as a 'community dictatorship' or can specialised evaluators retrieve proposals that have been forgotten and that they, as experts, think may help brand valuation? [Participant 13, Lecturers' group]

\section{Discussion}

It turned out that the Mentoring domain was considered the most interesting; in its most 
representative technological feature no unnecessary or missing functionalities were registered, and no functional obstacles were identified.

The Co-Design and Transfer domain was not considered a novelty (in fact, it embodies the role of an online community and social network dedicated to the brand), but revealed unexpected concerns with acknowledgment, authorship and content protection.

Finally, the Evaluation domain raised more questions than all the others, although participants recognised the advantage of being co-evaluators.

It appears that the issues of most concern to UA's employees and which they unanimously consider relevant are the involvement of middle and senior management (deans and provost team) and the recognition of authorship and protection of property contributions made in the PBC. Thus, despite its internal approach (which would require registration) and regarding the academic context of UA, with its somewhat weak social identity, a PBC aimed at holistically promoting the brand would need some sort of content protection.

Moreover, for such contributions to be published, employees would need to understand how their involvement in brand valuation would be acknowledged and whether their ideas are to be implemented or not. Besides manifestations of altruism, most individuals need to know that their participation will lead to certain individual or collective benefits in the short, medium or long term. In other words, the mere fact of publishing an artefact should be linked to some positive return, with some means of recognition from the community and from middle and top management being the first indication of consequence.

\section{A heuristic for brand artefact co-design}

Since we adopted UA's organisational context, there are no pretentions of 
generalisation. However, we can aim for transferability i.e., the ability to transfer results to other groups or contexts, in similar conditions (Mantzoukas, 2004).

We thus followed the conceptual framework of accessibility and universal design requirements proposed by Stephanidis et al. (1998): the proposal of general principles, for which some guidelines may be specified and from which a set of recommendations can be developed for each given context. Although they might not always be adaptable to all contexts, heuristics can be of great usefulness, sparing time spent analysing and evaluating all possibilities (Oliver and Roos, 2002).

Therefore, we have prepared a heuristic for a $B a$ aimed at brand artefact codesign, consisting of principles and guidelines, but without any recommendations, which would largely depend on each organisational context. The heuristic approach hereby aims to propose an intervention formula that maximises the likelihood of a specific effect associated with one or more events that may occur. The specific effect is brand valuation, boosted by an online PBC, driven by employees' co-designed contributions. Thus, the three domains of the interaction structure for brand artefacts codesign define the basic principles of such a formula:

(1) A PBC must include resources and must maximise events for brand learning and brand experimentation. In fact, it is with this in mind that employees begin a simultaneously cognitive and affective exploration of the $\mathrm{PBC}$ as a $B a$, through the exchange of information, the development of brand knowledge, and the opportunities for informal individual or collective co-design.

(2) A PBC must foster collaboration as well as the sharing and transfer of brand artefacts. Employees should be invited to contribute to brand design, proposing relatively tangible artefacts. Gamification is an essential condition for visual literacy to 
develop, so that creativity can occur. Collaboration may happen at any moment during the use of the PBC, individually or in collective situations.

(3) A PBC must explore the right to expression and the possibility of evaluating brand artefacts. Employees can and should evaluate brand artefacts published by their peers, in a transparent, informed and simplified way, thereby also contributing to brand knowledge, and potentially leading to the implementation of certain artefacts.

\section{Conclusions and further research}

Since internal and participatory design activities are still unknown or neglected in many organisations, this paper aims to draw brand designers and managers attention to the experiential perspective, which provides significant opportunities for co-design, and for the participation of employees in design processes.

By adopting strategies of brand artefact co-design, involving every employee, organisations must ensure a climate of creativity and of knowledge transfer/combination, which is evident to all. Reward systems should be designed and explained to those who will be sharing their individual knowledge with the organisational collective. Organisations should thus encourage employees to maximise their human capital, developing or optimising skills through participatory activities, involving themselves in pleasurable and meaningful experiences, after identifying and committing to the brand and the organisational identity.

The results of both the literature review and focus groups revealed that $\mathrm{CMC}$ can make a huge contribution in these participatory processes, particularly with the involvement of specific online communities that promote prosocial behaviours and allow the integration, conversion, sharing, reuse and evaluation of all the scattered 
brand-related information and knowledge. We have presented an interaction structure to support such processes and a heuristic to aid its implementation.

Due to the selection of a public academic context (with particular characteristics) as a case study, it is difficult to fully extrapolate these findings into broader universes or other institutional contexts. Thus, any attempt at generalisation could neither be linear nor direct, in any case. Transferability would be the right approach in order to apply the results obtained to other similar cases, such as other higher education institutions. Future research will include other organisational contexts, namely within a corporate context, where brand artefacts can constitute a fundamental business asset, which is not such a pertinent condition within the academic organisation eco-system. The academic workforce was excellent to test the collective design propensity and to what extent the organisation's collaborators would adopt it in their daily routines.

Nevertheless, we believe that, regarding human capabilities and overall engagement with the brand, it will not be very different if we approach a University with 1,600 employees or a medium to large standard corporate workforce. Naturally, such a company will need to fulfil certain requisites:

- Be open to collaboration, to co-design and to the participation of non-specialised individuals in highly specialised activities;

- Ensure an ecology of creativity and knowledge;

- Have one or more brands significant to its business(es);

- Hold a significant body of employees, which would justify the need of an online $B a$ and, therefore, ensure the sustainability of collective intelligence and its permanent relation to prospective innovative business intelligence. 
Finally, recognition, as a sign that the organisation is aware of its employees' contributions, will be the starting point for a conscious and informed participation process that is both interested and genuine. Thus, organisations should encourage employees to maximise their human capital, developing or optimising skills through participatory and 'gameable' activities, involving them in pleasurable and meaningful experiences, after which they can identify, learn and own the brand.

\section{Funding Acknowledgement}

For this research the first author was supported by a PhD scholarship, granted by the Portuguese Science and Technology Foundation, funded by the European Social Fund and by the III EU Support Framework (Grant No. SFRH / BD / 77659 / 2011).

\section{Acknowledgements}

The authors would like to acknowledge the focus groups participants for giving their time to discuss the theme and get involved in this study. Further, the authors would like to thank the editors and anonymous reviewers for their useful comments on earlier versions of this article.

\section{References}

Ackerman, L. D. 2000. Identity is Destiny: Leadership and the roots of Value Creation. San Francisco: Berrett-Koehler Publishers, Inc.

Alavi, M., and Leidner, D.E. 2001, " Review: knowledge management and knowledge management systems: conceptual foundations and research issues"', MIS Quarterly, 25(1), 107-36.

Arnhold, U. 2010. User Generated Branding: Integrating User Generated Content into Brand Management: Gabler Verlag. 
Barab, S. A., MaKinster, J. G., Moore, J. A., and Cunningham, D. J. 2001. "Designing and Building an On-line Community: The struggle to support Sociability in the Inquiry Learning Forum”. Educational Technology Research and Development, 49(4), 71-96.

Bjögvinsson, E., Ehn, P., and Hillgren, P.-A. 2012. "Design Things and Design Thinking: Contemporary Participatory Design Challenges”. DesignIssues, 28(3), 101-116.

Burmann, C., and Zeplin, S. 2005. "Building Brand Commitment: A behavioural approach to internal brand management". Journal of Brand Management, 12(4), 279-300

Buur, J., Ankenbrand, B., and Mitchell, R. 2013. "Participatory business modelling". CoDesign: International Journal of CoCreation in Design and the Arts, 9(1), 5571. doi:10.1080/15710882.2012.760609

Chong, M. 2007. "The role of internal communication and training in infusing Corporate Values and Delivering Brand Promise: Singapore Airlines' Experience". Corporate Reputation Review, 10(3), 201-212.

Feast, L. 2012. "Professional perspectives on collaborative design work". CoDesign: International Journal of CoCreation in Design and the Arts, 8(4), 215-230.

Fischer, G. 2007. Designing Socio-Technical Environments in support of Meta-Design and Social Creativity. Paper presented at the Computer Supported Collaborative Learning (CSCL '2007), Rutgers University, July pp. 1-10. Retrieved 14/03/2011 from http://13d.cs.colorado.edu/ gerhard/papers/CSCL-2007.pdf.

Fischer, G. 2009. "End-User Development and Meta-Design: Foundations for Cultures of Participation”. Lecture Notes in Computer Science, 5435/2009, 3-14.

Fischer, G., and Scharff, E. 2000. Meta-Design - Design for Designers. Paper presented at the 3rd International Conference on Designing Interactive Systems (DIS 2000), New York City (available in http://13d.cs.colorado.edu/ gerhard/papers/dis2000.pdf ).

Harris, P. 2007. "We the People: The importance of employees in the process of building customer Experience”. Journal of Brand Management, 15(2), 102-114. 
Hatch, M. J. 2007. Corporate Branding International Encyclopedia of Organization Studies. Thousand Oaks, CA: Sage Publications.

Ind, N. 2007. Living the Brand: How to transform every member of your organization into a Brand Champion (3rd ed.). London: Kogan Page.

Ind, N., and Bjerke, R. 2007. Branding Governance: a participatory approach to the brand building process. West Sussex: John Wiley \& Sons Ltd.

Jarvenpaa, S. L., and Staples, D. S. 2001. "Exploring perceptions of organizational ownership of Information and Expertise". Journal of Management Information Systems, 18(1), 151-183.

Jenkins, H. 2009. Confronting the Challenges of Participatory Culture - Media Education for the 21st Century. Chicago: The John D. and Catherine T. MacArthur Foundation.

King, C., and Grace, D. 2008. "Internal Branding: Exploring the employee's Perspective”. Journal of Brand Management, 15, 358-372.

Lélis, C. \& Mealha, Ó. 2014. Brand Experience Oriented Design Environments. BrandTrends, Journal of Strategic Communication and Branding, 6(6), April 2014, 6-18 (available online in http://www.brandtrendsjournal.com).

Lélis, C. \& Mealha, Ó. 2013. Sustainable Brands need Employees' Creativity, Proceedings of CMC 2013, 18th International Conference on Corporate and Marketing Communication, April 11-12, 2013, University of Salerno, Italy, ISBN: 9788890687365.

Lélis, C. \& Mealha, Ó. 2012. Learning the organizational brand with computermediated communication, Proceedings of IADIS International Conference eLearning 2012, 17-20 July 2012, Lisbon, Portugal, 493-496, ISBN: 978-9728939-71-7.

Mangold, W. G., and Miles, S. J. 2007. "The Employee Brand: Is yours an all-star?” Business Horizons, 50, 423-433.

Mantzoukas, S. 2004. "Issues of Representation within Qualitative Inquiry”. Qualitative Research Health, 14(7), 994-1007. 
Masino, G., and Zamarian, M. 2003. "Information technology artefacts as structuring devices in Organizations: Design, Appropriation and use Issues". Interacting with Computers, 15(5), 693-707.

McEnally, M., and de Chernatony, L. 1999. "The evolving nature of Branding: Consumer and Managerial Considerations". Academy of Marketing Science Review, 2, Available: http://www.amsreview.org/articles/mcenally02-1999.pdf.

McLoughlin, C., and Lee, M. 2007. Social software and participatory Learning: Pedagogical choices with technology affordances in the Web 2.0 Era. Paper presented at the Ascilite Singapore 2007, Singapore.

Merz, M. A., He, Y., and Vargo, S. L. 2009. "The evolving Brand Logic: a servicedominant logic perspective". Journal of the Academy of Marketing Science, $37(3), 328-344$.

Moingeon, B., Ramanantsoa, B. 1997. "Understanding Corporate Identity: the French school of thought". European Journal of Marketing, 31(5/6), 383-395.

Morgan, D. L. 1997. Focus Groups as Qualitative Research (2nd ed. Vol. 16). Thousand Oaks, CA: Sage Publications.

Morgan, G. 2006. Images of Organization. Thousand Oaks, CA: SAGE Publications.

Neto, R., and Choo, C. W. 2010. The Post Nonaka Concept of Ba: eclectic roots, evolutionary paths and future advancements. Paper presented at the ASIST 2010, Pittsburgh, PA.

Neumeier, M. 2006. The Brand Gap. Berkeley CA: New Riders.

Nonaka, I., and Konno, N. 2005. “The concept of 'Ba': building a foundation for knowledge creation". In I. Nonaka (Ed.), Knowledge Management: Critical Perspectives on Business and Management. Oxon: Routledge.

Nonaka, I., Konno, N., and Toyama, R. 2001. "Emergence of 'Ba'". In I. Nonaka \& T. Nishiguchi (Eds.), Knowledge Emergence - Social, Technical and Evolutionary Dimensions of Knowledge Creation. NY: Oxford University Press.

Nonaka, I., Reinmoeller, P., and Senoo, D. 1998. “The 'ART' of Knowledge: Systems to Capitalize on Market Knowledge". European Management Journal, 16(6), 673-684. 
Nonaka, I., and Takeuchi, H. 1995. The knowledge-creating Company - how japanese companies create the dynamics of Innovation. New York: Oxford University Press.

Oliver, D., and Roos, J. 2002. Organizational Heuristics: more than simple Rules. Paper presented at the 5th International Conference on Organizational Discourse. Kings College, London, UK. Retrieved from www.imagilab.org/pdf/wp02/WP13.pdf 23/03/2001.

Paulini, M., Murty, P., and Maher, M. L. 2013. "Design processes in collective innovation communities: a study of communication". CoDesign: International Journal of CoCreation in Design and the Arts, 9(2), 90-112. doi:10.1080/15710882.2012.716850

Pitt, L., Watson, R., Berthon, P., Wynn, D., and Zinkhan, G. 2006. “The Penguin's Window: Corporate Brands from an Open-Source Perspective". Journal of the Academy of Marketing Science, 34(2), 115-127.

Punjaisri, K., and Wilson, A. 2007. "The role of Internal Branding in the delivery of Employee Brand Promise". Journal of Brand Management, 15(1), 57-70.

Rafaeli, A., and Pratt, M. 2006. Introduction: Artifacts and Organizations: More than the tip of the Cultural Iceberg. In A. Rafaeli \& M. G. Pratt (Eds.), Artifacts and organizations: beyond mere symbolism. Mahwah, NJ: Lawrence Erlbaum Associates.

Reichwald, R., Möslein, K., and Piller, F. 2008. Creating Value Interactively Challenges for Company Management. In W. Buhse \& S. Stamer (Eds.), Enterprise 2.0: the art of letting go. Bloomington, IN: iUniverse, Inc.

Sanders, E. B.-N., and Stappers, P. J. 2008. "Co-creation and the new landscapes of Design”. CoDesign: International Journal of CoCreation in Design and the Arts, 4(1), 5-18.

Schein, E. H. 2010. Organizational Culture and Leadership (4th ed.). San Francisco, CA: Jossey Bass.

Schultz, M., and Hatch, M. J. 2006. A cultural perspective on corporate Branding - The case of LEGO Group. In J. E. Schroeder \& M. Salzer-Mörling (Eds.), Brand culture. Oxon: Routledge. 
Schultz, M., Hatch, M. J., and Ciccolella, F. 2006. Brand Life in Symbols and Artifacts: the LEGO Company. In A. Rafaeli \& M. Pratt (Eds.), Artifacts and Organizations. Mahwah, New Jersey: Lawrence Erlbaum.

Stephanidis, C., Akoumianakis, D., Sfyrakis, M., and Paramythis, A. 1998. Universal Accessibility in HCI: Process-oriented design guidelines and tool requirements. Paper presented at the 4th ERCIM Workshop on User Interfaces for All, Stockholm, Sweden.

Tee, M.Y. 2005, “Tacit knowledge in a e-learning environment: a naturalistic study", $\mathrm{PhD}$ dissertation, University of Kansas, Lawrence, KS.

Thorpe, A., and Gamman, L. 2011. "Design with society: why socially responsive design is good enough". CoDesign: International Journal of CoCreation in Design and the Arts, 7(3-4), 217-230. doi:10.1080/15710882.2011.630477

Vaajakallio, K., and Mattelmäki, T. 2014. "Design games in codesign: as a tool, a mindset and a structure”. CoDesign: International Journal of CoCreation in Design and the Arts, 10(1), 63-77.

Vilnai-Yavetz, 1., and Rafaeli, A. 2006. Managing Artifacts to avoid Artifact Myopia. In A. Rafaeli \& M. G. Pratt (Eds.), Artifacts and organizations: beyond mere symbolism. Mahwah, NJ: Lawrence Erlbaum Associates.

Zichermann, G., and Cunningham, C. 2011. Gamification by Design: Implementing Game Mechanics in Web and Mobile Apps. Sebastopol, CA: O'Reilly Media. 
Table 1. Detail Questions and Analysis Codes.

\begin{tabular}{|l|l|}
\hline \multicolumn{1}{|c|}{ Guidance Question and Detail Questions } & \multicolumn{1}{|c|}{ Analysis Codes } \\
\hline Would it make sense to implement an online resource based on the presented? & \\
\hline What is the possibility of implementing a PBC at the University of Aveiro? & PBC Implementation \\
\hline What would be the advantages for the University of Aveiro and its employees? & Advantages \\
\hline What features seem to be unnecessary? & Unnecessary Features \\
\hline What features might be missing? & Missing Features \\
\hline What kind of obstacles could derail such resource? & Obstacles \\
\hline Personally, what would it take you to participate in such a platform? & Propensity \\
\hline
\end{tabular}


Figure 1. - The three main domains of the interaction structure for brand artefact codesign.

Figure 2. - The iterative structure of the $B a$ for brand artefact co-design.

Figure 3. - The process of Focus Groups content analysis. 